\title{
Study on the Microstructure of Goose Feather
}

\author{
Kaili Chen ${ }^{1}$,Yiping Yin, Xiaxin Xu, Shu Yang ${ }^{* 1, a}$, \\ ${ }^{1}$ Nanhu College, Jiaxing University, Zhejiang, Jiaxing, 314000, China \\ ayangshu5678@163.com
}

\begin{abstract}
Keywords: Goose Feather, Microstructure, Light, Structural Color
Abstract. In nature, many organisms produce colorful color. From the colored principle, its color may divide into chemistry color and the physical color generally. Many plants and animals color in nature is not derived from the pigment, but from the interaction of light and surface. To further understand the rules of color rendering in the nature, the structure of the feather samples were observed by optical microscopy and scanning electron microscopy (SEM). Colored feather consists of many barbules and is closely arranged. Pores between each barbule have different sizes, showing a grid shaped structure. Due to the different structure of the cycle and the absorption of visible light at different wavelengths, the feathers show a different color.
\end{abstract}

\section{Introduction}

In nature, many organisms produce colorful color. From the colored principle, its color may divide into chemistry color and the physical color generally. Chemical color refers to the color produced by the pigment. From the learning and understanding of nature, many animals and plants color is not derived from the pigment, but from the interaction of light and surface, such as goose feather, peacock feathers etc.

The structure color is the most common in our daily life. Sunny blue sky is due to Rayleigh scattering [1]. The oil stains on the surface of the water produced a color because of the thin film interference, and rainbows are colorful because of the index, and so on. In general, the structure color is produced by the interaction between light and microstructure, and the optical effects of refraction, diffraction, reflection, scattering and interference [1-4]. When the number of layers increases and the coherence becomes stronger, the light of a certain band is completely reflected, which forms a thin film interference [5]. The photonic crystal is a periodic dielectric structure with photonic band gap [6]. Inspired by this, this research attempts to study the of rule color rendering in nature. Its significance lies in the use of bionics method, and its color principle is introduced to color textile fabric design, so as to realize the color of fabric dye [7].

In this experiment, the sample is goose feather. When studying the optical structure of colored feathers, 3 levels can be observed. Different characteristics can be seen at each level. The first level is visible to the macrostructure by naked eye. The second level is overall plumage arrangement structure under the optical microscope. The third level can be seen under SEM, including microstructure, morphology, distribution on the surface.

\section{The macrostructure of the goose feather with the naked eye}

The sample is goose feather. Goose is believed to be the first domesticated poultry, which comes from the wild goose or grey goose. European goose from the grey goose, and Chinese goose from swan goose.

By macroscopic observation, macrostructure is shown in Fig. 1. 


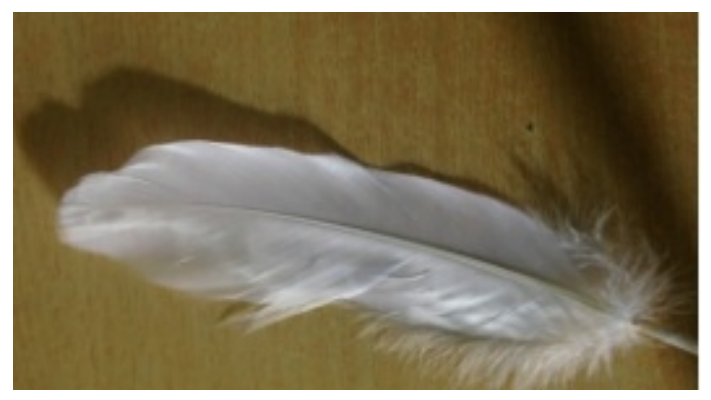

Fig. 1 Goose feather

As shown in Fig. 1, feather on the surface is white, and the density of hair is generally very close.

\section{The microstructure of the goose feather under optical microscope}

The optical microscope test is to have a whole understanding of the feather arrangement and distribution, and have a preliminary understanding of the size, shape, appearance and structure etc. In the optical microscope, it can clearly see the different display space and the color of feathers arranged inside.

Laboratory equipment. The laboratory equipment is fiber fineness instrument microscope. The select multiple magnification is $50 \times 10$ times.

Experimental process. Turn on the computer and the microscope switch. Open the fiber fineness instrument software and the mouse click on the window of the work bar, "a collection of". Adjust microscope illumination light source to make sample brightness moderate. Rotary converter, first with the low power of observation, adjusting the coarse quasi focus spiral. When adjusting, the objective lens is adjusted to the lowest, and then gradually adjusted. This prevents the objective lens from contacting the sample, which is easy to destroy the sample or to smear the objective. When the search to the image, change the fine focusing on spiral and adjust the focus until you see the clear picture. Choose high power observation. The choice of the test is 50 x 10 times. Adjust the fine quasi focal helix and the focal length until you see a clear picture, so that the image achieve the best clear results. Then take pictures of storage.

Experimental result. Fig. 2 shows a single feather. Fig. 3 shows the whole feather.

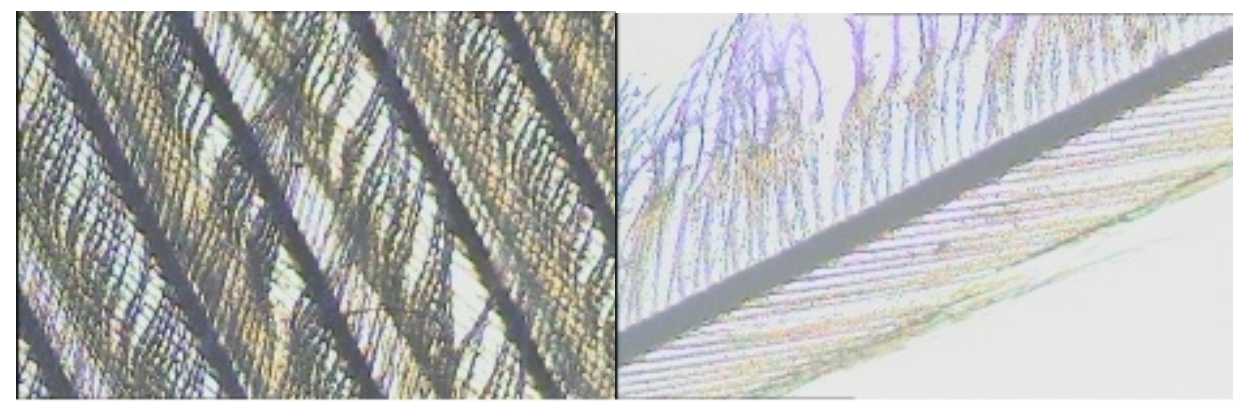

Fig. 2 A single feather

Fig. 3 The whole feather

Further amplification observed that pores between each barbule have different sizes. Owing to the transmissibility of the light, it shows a different color. In Fig. 2 and Fig. 3, the color change owing to the change of pores. There is a periodic distribution between each barbule, and the average diameter is about 200 380 um.

\section{The microstructure of the goose feather under SEM}

From the ordinary microscope test results, we have a general understanding of the feather. However, to reach the aim of studying and analyze the effect of feather microstructure on visible light, the 
magnification of an ordinary microscope is far from enough, which requires us use more microscopic method and test apparatus.

Laboratory equipment. This experiment use QUANTA-250 scanning electron microscope, which has more accurate and microscopic description of goose feather.

Sample production. Skim: first, sample with normal saline $(0.65 \% \mathrm{NaCl}$ solution $)$ repeatedly washed and fixed with $3 \%$ glutaraldehyde. In ultrasonic cleaner processing (power 50W) 1-4min, you can wash away the dirt attached to the surface. Ether can be used to skim for the surface mucus, fat and protein content. Samples will be placed in containers with ether soak for 10 minutes.

Dehydration: after taking out the sample, the sample was taken out and dehydrated with ethanol. The ethanol concentration in this test was 40\%, 50\%, 70\%, and pure ethanol. Each phase was soaked for 10 minutes, and the samples were dried by natural drying method.

Sticky table and spray gold: dry samples with double-sided adhesive. Pay attention to the upper surface of the sample when pasting. In order to make the metal ions uniformly sprayed on the surface of the sample, it should be used in a vacuum of $1 \times 10^{5} \mathrm{MPa}$ or so, with a 40 degree rotating spray plating or sputtering with an ion sputtering apparatus. The gold plating thickness of the sample should be between 15 and 20nm. If the sample surface has a lot of villi, spines, scales, it should be put into the $1 \%$ potassium iodide solution for 2 days. After removal, clean with distilled water and dry naturally. And then spray gold.

Experimental result. Adjust the observation point of view, to find a complete, clear structure of the scale, and adjust the different amplification. Finally, the test results are shot and stored. The sample was magnified 100 times, 500 times, 1000 times, 5000 times to observe the feather samples. The experimental results are shown in Fig. 4.

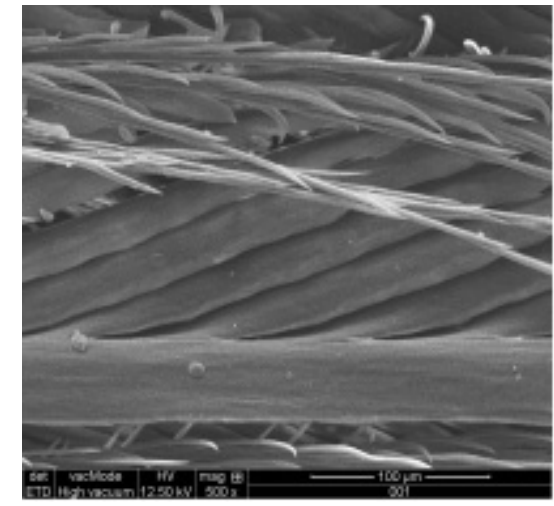

(a)

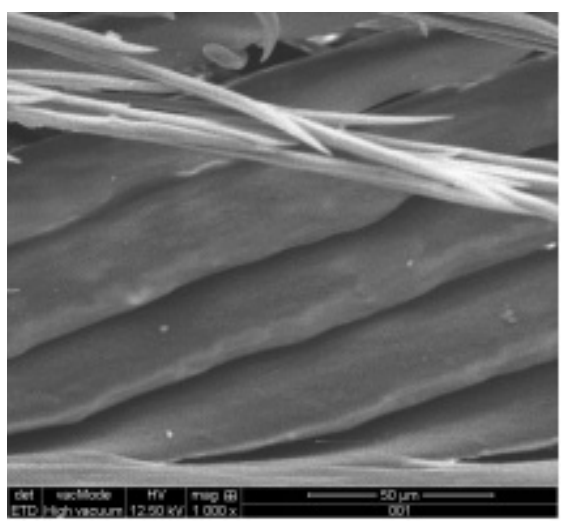

(c)

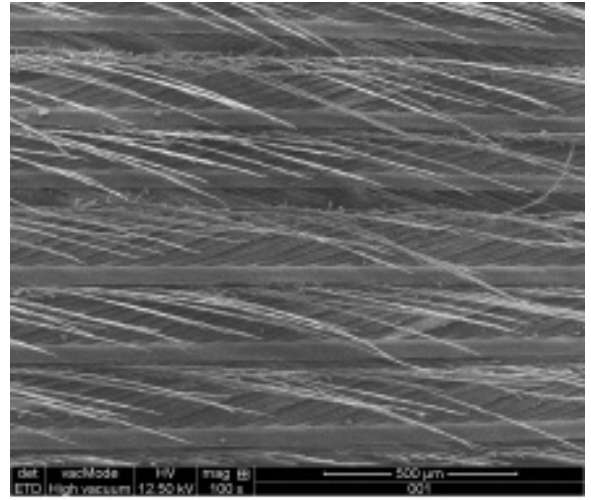

(b)

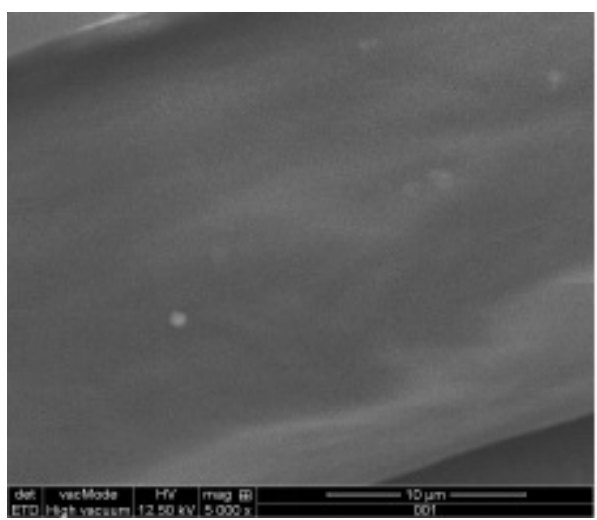

(d)

Fig. 4 Experimental results under SEM with different magnifications. The magnifications are: (a) 100 times, (b) 500 times, (c) 1000 times, (d) 5000 times. 
As Figure 4 shows, goose feather is constituted by a plurality of barbules. Periodic structures are line shaped in each barbule. The diameter is about 190 230 um. Each barbule has a gap overlap with regular distribution.

\section{Conclusions}

Through the observation and analysis on the microstructure of goose feather surface, feather arranged closely and its surface like fish scales as piece covered with another piece. They linked by a certain amount of adhesive proteins. Feather consists of many barbules, and each barbule has cycle distribution. Each has a gap overlapping, showing a grid structure in the whole surface. Pores between each barbule have different sizes. Due to the different structure of the cycle and the absorption of visible light at different wavelengths, the feathers show a different color. The microstructure of feather was studied, hoping for the dye chromophore to provide new ideas for color fabrics.

\section{Acknowledgements}

This work was financially supported by the National Natural Science Foundation of China (Grant No. 51403078), China Scholarship Council(Grant No.201508330017) and Zhejiang province education department(Grant No.Y201226223), and SRT project of Jiaxing University(851715079).

\section{References}

[1] Zhiwu Han, Liyan Wu , Zhaomei Qiu and Luquan Ren. The micro structure and color purple ring Butterfly Scales [J]. Science Bulletin (2008) (In Chinese)

[2] Wei Yuan. Preparation and optical properties of structural color fiber or fabric [D]. Soochow University (2014) (In Chinese)

[3] Haiwei Yin. Study on the synthesis of mechanism and system structure of color couplers [D]. Fudan University (2013) (In Chinese)

[4] Wenjun $\mathrm{Wu}$. Study on optical properties and color changing mechanism of butterfly wing micro nano structure [D]. Huazhong University of Science and Technology (2012) (In Chinese)

[5] Parker A. R, Welch V. L, Driver D, Martini N, Structural colour: Opal analogue discovered in a weevil [J], Nature (2003), 426, 786

[6] Joannopoulos J. D, Meade R. D, Winn J. N, Meade R. D, Photonic crystals:molding the flow of light (second edition) [M], NJ: Princeton Univ. Press (1995)

[7] "Experimental study of Papilio maackii wing structure photochromic mechanism", Chinese Science Series E, Vol. 37 (2007), p. 6 (In Chinese) 